\title{
Single qubit from two coupled quantum dots: An approach to semiconductor quantum computations
}

\author{
Xin-Qi Li* and Yasuhiko Arakawa \\ Research Center for Advanced Science and Technology, University of Tokyo, 7-22-1 Roppongi, Minato-ku, Tokyo 106, Japan
}

(Received 24 January 2000; published 7 December 2000)

\begin{abstract}
A scheme to possibly implement the quantum computer is proposed based on the idea to build up the single qubit from two coupled quantum dots. The spatial separation of the two logic states of the qubit can greatly reduce the decoherence time, which allows one to perform the coherent quantum logic operations at a fairly high ratio. Together with the realization of the universal quantum logic gates, and the proper scheme of quantum measurement, the present proposal can satisfy all the essential criteria for quantum computing.
\end{abstract}

DOI: 10.1103/PhysRevA.63.012302

PACS number(s): 03.67.Lx, 73.61.-r, 89.70.+c

Quantum computers (QCs) have generated broad interest in the past years $[1-3]$. The existing QC proposals include utilizing electrodynamic cavities [4], ion traps [5], nuclear and electron spins [6-8], and superconductor Josephson junctions [9-11]. To the end of large-scale integration, the solid-state implementations, especially those based on semiconductor nanostructures by regarding the rapid progress in this field, would be extremely desirable. In this paper, we propose a scheme to reduce the decoherence of the charge degrees of freedom in quantum dots (QDs), and implement the universal quantum gates for a QC. We suggest building up a single qubit from two coupled QDs, and operating the qubit rotations by the quantum cascade lasers [12]. It will be demonstrated that the spatial separation of the logic states can largely reduce the decoherence, and the decoherence time can exceed the gate switching time by a ratio, for example, higher than 5000, which sensitively depends on the coupling strength between the two logic states.

Qubits (highly coherent two-level systems), and the universal quantum gates based on them (i.e., the two-qubit controlled-NOT gate in combination with the single qubit rotations), set up the physical foundation for a QC. Based on semiconductor QDs, in Fig. 1 we show the central idea of our proposal for constructing the qubits. Instead of making a qubit from a single quantum dot, we build up a single qubit from two coupled quantum dots with different sizes, which contains one electron in it. Each qubit is isolated from the other by a wide barrier and by the Coulomb blockade effect. Under this construction, it is expected that the spatial separation between the qubit logic states $|0\rangle$ and $|1\rangle$ is able to remarkably reduce the decoherence. We noted that a similar idea of this spatial separation has been successfully exploited by Capasso and co-workers [12] in their famous cascade laser structures, where the spatial separation of the two coupled quantum wells greatly reduced the relaxation of carriers from the higher state to the lower one, thus realizing the population inversion in their cascade laser structures. Below we analyze the qubit decoherence and demonstrate the universal quantum gate operations.

\footnotetext{
*Present address: Department of Chemistry, Hong Kong University of Science and Technology, Clear Water Bay, Kowloon, Hong Kong, China. Email address: xqli@ chsg4.ust.hk
}

Specifically, consider a coupled-quantum-disk geometry shown in Fig. 1(a), and assume a parabolic potential for the lateral confinement (for more detailed descriptions, see the caption of Fig. 1). Our quantitative estimate will rely on a model system (GaAs based structure) with effective electron mass $m^{*}=0.067 m_{e}$ ( $m_{e}$ is the bare electron mass), and the material relative dielectric constant $\epsilon / \epsilon_{0}=12.9$ where $\epsilon\left(\epsilon_{0}\right)$ is the material (vacuum) dielectric constant. We assume the effective well depth $V_{0}=600 \mathrm{meV}$, and the quantum disk sizes are designed so as to obtain a large logic level spacing;

\section{a}

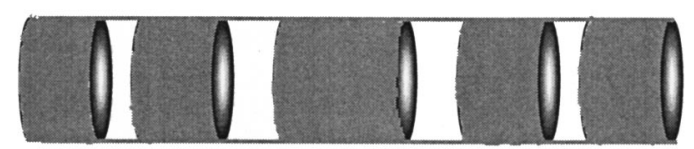

b

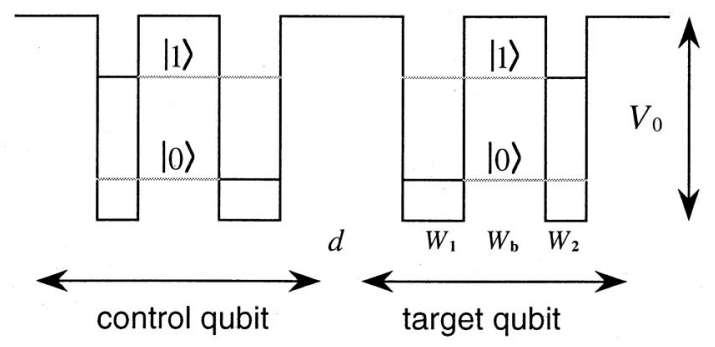

FIG. 1. Qubits based on coupled QDs. (a) Schematic geometry of the coupled QDs, i.e., quantum disks, where the parabolic potential for the lateral confinement is assumed, $V(x, y)=\left(m^{*} / 2\right) \omega_{0}^{2}\left(x^{2}\right.$ $\left.+y^{2}\right)$, which results in a lateral level structure $\epsilon_{x, y}=\left(n_{x}+n_{y}\right.$ $+1) \hbar \omega_{0}$ and the corresponding state $\phi_{n_{x} n_{y}}(x, y)$. (b) Band offset of different materials in the $z$ direction results in an effective onedimensional quantum wells. We employ two coupled QDs to build up a single qubit, and each qubit, containing one electron in it, is isolated from the other by a wide barrier and by the Coulomb blockade effect. For a single qubit, the two logic states $|0\rangle$ and $|1\rangle$ localize mainly in each different dot. The $z$ component of the one-particle eigenstate is denoted by $\chi_{n}(z)$, and the logic states are $|0\rangle$ $=\left|\phi_{00}(x, y)\right\rangle\left|\chi_{0}(z)\right\rangle$ and $|1\rangle=\left|\phi_{00}(x, y)\right\rangle\left|\chi_{1}(z)\right\rangle$, respectively. The Coulomb coupled two qubits shown in this figure enable us to realize the controlled-NOT quantum gate. 
e.g., $\Delta E=225 \mathrm{meV}$ if we choose $W_{1}=5.3 \mathrm{~nm}, W_{2}$ $=2.8 \mathrm{~nm}$, and a barrier width $W_{b}=3 \mathrm{~nm}$. The lateral parabolic confinement is assumed to result in a lateral level spacing $\hbar \omega_{0}=25 \mathrm{meV}$, which is achievable in the updated laboratory. Before demonstrating the gate operations, the most significant procedure is to investigate the qubit decoherence, which in our case intrinsically originates from the spontaneous light emission, and the nonradiative electron-phonon interactions. We estimate them in order in the following:

(i) Spontaneous emission. Under the dipole approximation, based on the Fermi golden rule, we are able to derive the following expression for the spontaneous emission rate:

$$
\tau_{R}^{-1}=\frac{e^{2}}{2 \pi \epsilon_{0} \hbar} \sqrt{\frac{\epsilon}{\epsilon_{0}}}\left(\frac{\Delta E}{\hbar c}\right)^{3}|\langle 0|z| 1\rangle|^{2},
$$

where $e$ is the electron charge, $c$ the light speed, and $\Delta E$ the level spacing between $|0\rangle$ and $|1\rangle$. We also assume that the surrounding medium of the quantum dots has the same dielectric constant as the quantum dots. Note that the dipole operator only couples the logic state $|1\rangle$ to $|0\rangle$, and does not couple $|1\rangle$ to other lateral quantum states resulting from $|0\rangle$. Further, in Eq. (1) only the $z$-polarization component has a contribution. The lateral $x$ - and $y$-polarization components vanish because of the factorized feature of the wave functions (see caption of Fig. 1).

(ii) Electron-LA-phonon scattering. Due to the extremely fast decrease of the LA-phonon scattering rate with the increase of the electronic state energy difference, we consider the dominant contribution of scattering from the logic state $|1\rangle=\left|\phi_{00}(x, y)\right\rangle\left|\chi_{1}(z)\right\rangle$ to the nearest-neighbor lower state $|N ; 0\rangle \equiv\left|\phi_{n m}(x, y)\right\rangle\left|\chi_{0}(z)\right\rangle$, where $N=n+m, n$, and $m$ are the corresponding lateral quantum numbers. At zero temperature, the LA-phonon scattering rate is given by the golden rule as

$$
\frac{1}{\tau_{\mathrm{LA}}}=\frac{2 \pi}{\hbar} \sum_{q}\left|M_{q}\right|^{2} \delta\left(\Delta E_{1, N}-\hbar \omega_{q}\right),
$$

with $M_{q}=D \sqrt{\hbar q / 2 \rho v_{s} V}\left\langle N ; 0\left|e^{i \mathbf{q} \cdot \mathbf{r}}\right| 1\right\rangle$. For GaAs material, the deformation potential constant $D=6.8 \mathrm{eV}$, the density $\rho=5.36 \mathrm{~g} / \mathrm{cm}^{3}$, and the sound velocity $v_{s}=5.15$ $\times 10^{5} \mathrm{~cm} / \mathrm{sec}$. $V$ is the system volume and will be canceled by the wave-vector $\mathbf{q}$ summation. In Eq. (2) $\Delta E_{1, N}$ is the energy difference between the states $|1\rangle$ and $|N ; 0\rangle$, and is assumed to be the lateral level spacing $\Delta E_{1, N} \simeq \hbar \omega_{0}$ $=25 \mathrm{meV}$.

(iii) Electron-LO-phonon scattering. In quantum dots, due to the discrete nature of the energy levels together with the very weak energy dispersion of the LO phonons, a simple consideration on the basis of energy conservation predicted a vanished LO-phonon scattering rate, unless the electron level spacing almost equals the LO-phonon energy. This is referred to as the phonon bottleneck $[13,14]$. Recently, we showed that due to the finite lifetime of the LO phonons this conclusion should be modified [15]. The basic idea is as follows: For the coupled electron-LO-phonon system, similar to the coupled atom-photon system in an optical microcavity, the quantum transition would result in a repeated energy ex- change between the electron and the LO phonons if the electron level spacing is not far, but can be quite different from the LO-phonon energy. Owing to the decay of LO phonons into acoustic phonons [16], the electron's energy will be dissipated away eventually. In the present case, to estimate the dephasing time of the logic state $|1\rangle$ subjected to the LOphonon scattering, we consider the lower electronic state being separated from $|1\rangle$ by an energy that is closest to the LO-phonon energy, which makes the dominant contribution to the dephasing time. Neglecting the dispersion of the LOphonon energy, a Wigner-Weisskopf treatment leads to an analytical expression for the dephasing time as [15]

$$
\tau_{\mathrm{LO}}=2 \pi \hbar /\left(\Gamma-2 \beta_{2}\right),
$$

where $\Gamma \simeq 0.6 \mathrm{meV}$, associated with the LO-phonon lifetime $\sim 7$ ps [16], and $\beta_{2}$ is the absolute value of the imaginary part of $\beta . \beta$ is obtained from [15]

$$
\beta^{2}=\left[\gamma^{2}+\left(\Delta^{2}-\Gamma^{2}\right) / 4\right]+i \Gamma \Delta / 2 .
$$

$\Delta$ is the detuning energy between the electronic two-level spacing and the LO-phonon energy. In our estimate we set $\Delta \simeq 10 \mathrm{meV} . \gamma$ is the coupling strength between the reduced electronic two-level system and the LO phonons, and can be calculated from [15]

$$
\gamma^{2}=\sum_{q}\left|V_{q}\right|^{2}\left|\left\langle N ; 0\left|e^{i \mathbf{q} \cdot \mathbf{r}}\right| 1\right\rangle\right|^{2}
$$

where $\left|V_{q}\right|^{2}=4 \pi \alpha \sqrt{\hbar / 2 m^{*} \omega_{\mathrm{LO}}}\left(\hbar \omega_{\mathrm{LO}}\right)^{2} / q^{2} V$, characterizing the Fröhlich interaction strength. For GaAs material, the dimensionless polaron constant $\alpha=0.07$, and the LO-phonon energy $\hbar \omega_{\mathrm{LO}}=36 \mathrm{meV} . V$ is the system volume as above.

In the following, we analyze the gate operations in the presence of the above decoherence sources. First, for the single qubit rotation, we propose an optically driven approach. Under the rotation-wave approximation, the action of the laser on the qubit is described by the Hamiltonian

$$
\mathcal{H}_{I}=\hbar g\left(a^{\dagger} \sigma^{-}+a \sigma^{+}\right)
$$

where $a\left(a^{\dagger}\right)$ annihilates (creates) a photon, and Pauli matrices $\sigma^{-}$and $\sigma^{+}$describe the quantum transition between $|1\rangle$ and $|0\rangle$. The interaction strength $g$ $=e|\langle 0|z| 1\rangle| / 2 \hbar \sqrt{\hbar \omega_{f} / \epsilon V}$, if the laser field is polarized along the $z$ direction, where $\hbar \omega_{f}$ is the photon energy and $V$ the volume occupied by the laser mode. Under the action of the laser, the coupled electron-photon system undergoes the well-known Rabi oscillations. At zero detuning ( $\hbar \omega_{f}$ $=\Delta E$ ), the Rabi frequency is $\Omega=2 g \sqrt{n+1}$, where $n$ is the photon number of the laser field. Accordingly, the gate operation speed is determined by the time scale $\tau_{G}=\pi / \Omega$, with

$$
\Omega=\frac{e}{\hbar} \sqrt{\frac{\varepsilon_{f}}{\epsilon}}|\langle 0|z| 1\rangle|,
$$

where $\varepsilon_{f}=(n+1) \hbar \omega_{f} / V$ is approximately the field energy density if $n \gg 1$. In our estimate, we adopt the well-known quantum cascade laser parameter [12], i.e., the laser action 
TABLE I. Qubit decoherence and switching times. The qubit decoherence, characterized here by the spontaneous emission lifetime $\tau_{R}$, and the LA (LO) -phonon scattering lifetime $\tau_{\mathrm{LA}}\left(\tau_{\mathrm{LO}}\right)$, is reduced remarkably by increasing the spatial separation between $|0\rangle$ and $|1\rangle$. This significant observation enables us to achieve a high gate operation ratio due to the fact that the decoherence rate and the optical-driving Rabi transition speed are reduced in different scales with increasing $W_{b}$.

\begin{tabular}{ccccc}
\hline \hline$W_{b}(\mathrm{~nm})$ & $\tau_{R}(\mathrm{msec})$ & $\tau_{\mathrm{LA}}(\mathrm{sec})$ & $\tau_{\mathrm{LO}}(\mu \mathrm{sec})$ & $\tau_{G}(\mu \mathrm{sec})$ \\
\hline 3.0 & $1.6 \times 10^{-4}$ & $1.0 \times 10^{-4}$ & $9.6 \times 10^{-4}$ & $1.1 \times 10^{-4}$ \\
5.0 & $1.7 \times 10^{-3}$ & $3.6 \times 10^{-3}$ & $1.2 \times 10^{-2}$ & $2.8 \times 10^{-4}$ \\
7.0 & $2.1 \times 10^{-2}$ & 0.13 & 0.16 & $9.6 \times 10^{-4}$ \\
10.0 & 0.93 & 2.3 & 8.0 & $6.0 \times 10^{-3}$ \\
12.0 & 11.8 & 44.8 & 106.5 & $2.3 \times 10^{-2}$ \\
\hline \hline
\end{tabular}

with an output peak power of $\sim 8 \mathrm{~mW}$, which can provide a laser intensity $\varepsilon_{f} \simeq 10^{-3} \mathrm{~mJ} / \mathrm{cm}^{3}$ if the laser is focused onto an area of $\sim 100 \mu \mathrm{m}^{2}$.

To carry out the quantitative result, we approximately calculate the wave functions by isolating the concerned qubit from other qubits, and assuming infinitely high barriers on the two sides, while keeping the middle-standing barrier height as $V_{0}$. This procedure simplifies the numerical calculations, but does not affect the physical conclusions. In Table I, we present the estimated results for the various relaxation times of state $|1\rangle$, and the gating time of the qubit. In the presence of multiple relaxation channels, the decoherence time $\tau_{d}$ is obtained simply by the inverse summation law, $1 / \tau_{d}=\Sigma_{j} 1 / \tau_{j}$, where $\tau_{j}$ stands for the spontaneous emission lifetime $\tau_{R}$, and the LA (LO) phonon scattering lifetime $\tau_{\mathrm{LA}}\left(\tau_{\mathrm{LO}}\right)$, respectively. We observe that the spatial separation of the two logic states can efficiently reduce the decoherence. Interestingly and importantly, we find that the spatial separation would increase the ratio $\tau_{d} / \tau_{G}$, owing to the fact that each relaxation time becomes longer more rapidly than the gating time, as $W_{b}$ increases. The ratio $\tau_{d} / \tau_{G}$ is the key quantity for quantum computing, since it characterizes how many gate operations can be performed during the coherent time. We have noted that the ion trap QC can reach a gate operation ratio of $\sim 10^{4}[5]$, and the Josephson junction QC can reach $\sim 4000$ [9]. In our case, we can achieve a similar gate operation ratio; for example, $\tau_{d} / \tau_{G}>5000$ when $W_{b}$ is larger than $12 \mathrm{~nm}$. In principle, we can achieve an even higher ratio $\tau_{d} / \tau_{G}$ (by several orders of magnitude), if we increase $W_{b}$ further. We suggest that in practice this technique should be utilized thoroughly to improve the gating ratio. Other approaches to improve $\tau_{d} / \tau_{G}$ include, for example, enhancing the laser strength, and increasing the lateral confinement (i.e., fabricating smaller QDs). For ultrasmall QDs with considerably large lateral level spacing, the phonon dephasing mechanism will be largely suppressed, and the dominant dephasing comes from the spontaneous emission. In this regime, the gating ratio can be as high as $\sim 5 \times 10^{5}$ from Table I.

Next, consider the two-qubit operation schematically shown in Fig. 1(b). The charge states in the two qubits are in a fairly spatial separation, since the quantum tunneling be-

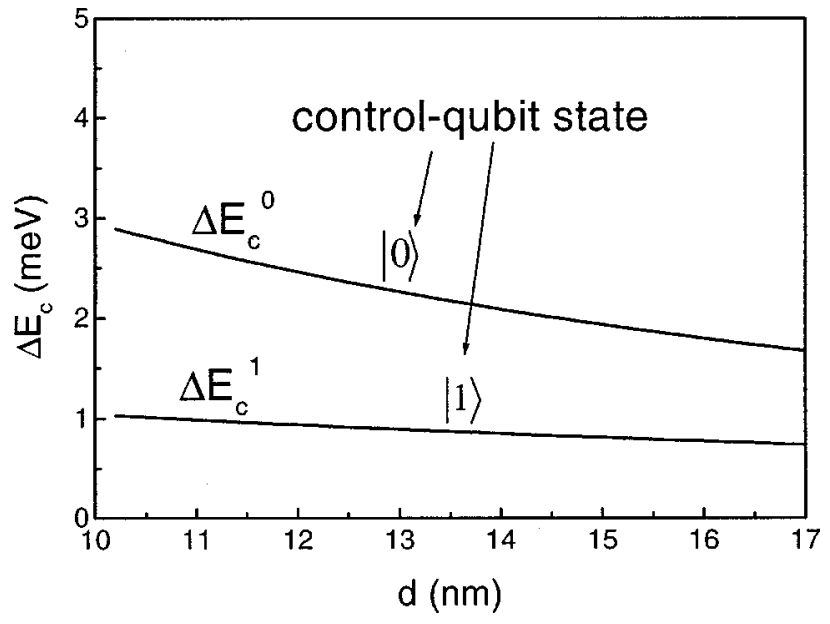

FIG. 2. Change of energy-level spacing of the target qubit, which depends on the control-qubit states $|0\rangle$ and $|1\rangle$, due to the different Coulomb interaction strengths, where we assume $W_{b}$ $=12 \mathrm{~nm}$, i.e., the spatial separation between the two dots in a single qubit.

tween the two qubits is suppressed by the middle-standing thick barrier and the Coulomb blockade effect. Due to the long-range Coulomb interaction between the control qubit and the target qubit, the charge states should, in principle, be solved self-consistently. For simplicity, but not losing the central physics, we treat this problem on the level of the lowest perturbation theory, namely, regarding the Coulomb interaction as perturbation, and using the zeroth-order wave function to estimate the first-order energy shifts. In the context of the controlled-NOT gate, consider the energy shift of the target qubit in state $|i\rangle$, due to the Coulomb perturbation from the control qubit in state $|j\rangle$

$$
E_{c}^{i j}=\left\langle i\left|H_{j}^{\prime}(\mathbf{r})\right| i\right\rangle
$$

where the Coulomb perturbation Hamiltonian $H_{j}^{\prime}(\mathbf{r})$ $=\int d^{3} \mathbf{r}^{\prime} e^{2}\left|\left\langle\mathbf{r}^{\prime} \mid j\right\rangle\right|^{2} / 4 \pi \epsilon\left|\mathbf{r}-\mathbf{r}^{\prime}\right|$. Depending on the controlqubit state in $|0\rangle$ or $|1\rangle$, the Coulomb interaction induced change of the level spacing of the target qubit is $\Delta E_{c}^{0}=E_{c}^{00}$ $-E_{c}^{10}$, or $\Delta E_{c}^{1}=E_{c}^{01}-E_{c}^{11}$. Figure 2 shows the calculated result. To implement the controlled-NOT gate, let us choose a laser frequency in resonance with the target-qubit level spacing corresponding to the control qubit in state $|1\rangle$. Accordingly, the target qubit flips between $|0\rangle$ and $|1\rangle$ under the laser action, if the control qubit is in $|1\rangle$; otherwise, the target-qubit state remains unchanged, if the control qubit is in $|0\rangle$. To avoid the control qubit responding to the laser pulse, in practice one may design the QD sizes of the control qubit being slightly different from the ones of the target qubit, which would result in a different level spacing for the control qubit, thus being in off-resonance with the laser frequency. In this way, we can implement the quantum controlled-NOT gate. Combining it with the single qubit rotations, the universal quantum gates for a $\mathrm{QC}$ are thus realized.

We emphasize here that the conditional transition tuning energy difference (CTTED) of the target qubit is as large as 


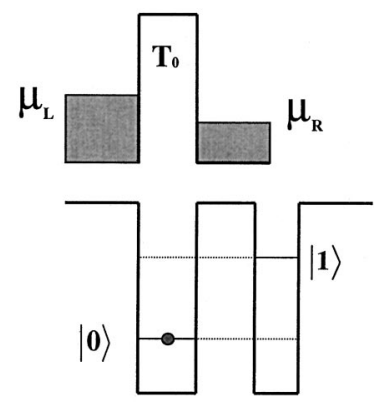

(a)

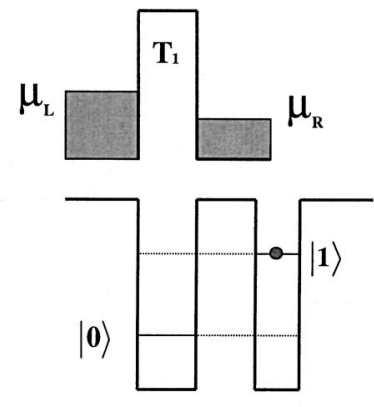

(b)
FIG. 3. Quantum measurement on the qubit state, performed by a quantum point contact or single-electron transistor. The transmission coefficient through the measuring device depends on the electron occupation in the left dot (a) or in the right dot (b).

about $1 \mathrm{meV}$ (see Fig. 2), which is much larger than the qubit-laser field coupling strength, thus implying a precise conditional transition control. Note that in the well-known trapped-ion quantum computer [5], the center-of-mass (c.m.) vibration frequency is about $50 \mathrm{KHz}$, corresponding to a phonon energy of about $10^{-4} \mathrm{meV}$, which plays the role of conditional transition control. Though this CTTED is extremely small, the conditional transition control can be performed with high accuracy, since the ratio of the control detuning energy and the system-optical field coupling strength is large. (Note that the weak coupling of the system with the driving laser field only results in a slow Rabi oscillation with period about $35 \mathrm{~ms}$, corresponding to an energy scale of $10^{-10} \mathrm{meV}$.)

To complete the proposal for a QC, we need to identify the quantum measurement; namely, after computation, we must be able to read out the qubit states (i.e., the result). For our structure, this can be accomplished by coupling the measured qubit to a quantum point contact (QPC) or a singleelectron transistor (SET), the latter playing the role of a measuring device, see Fig. 3. During quantum computing, the QPC (SET) is kept in a zero-current state, which has an unimportant but overall capacitance effect on the qubit. To perform a readout measurement, the transport voltage is turned on across the QPC (SET). Consequently, the quantum coherence of the measured qubit is destroyed, and the qubit state collapses into one of the two logic states, $|0\rangle$ or $|1\rangle$. Since a different qubit logic state would induce a distinct electro potential on the QPC (SET), i.e., affect the transmission coefficient, the dissipative current through the QPC (SET) carries the information of the charge state of the mea- sured qubit. The detailed dynamics for this kind of quantum measurement has been carried out recently [17-19].

So far, our analysis is based on a certain ideal situation, where we have shown that the proposed semiconductor QC has no intrinsic difficulties. In practice, the major challenge comes from the capacity of fabricating high-quality quantum dots. The moderate irregularity of the QD sizes or shapes due to the imperfect fabrications seems endurable, since it simply modifies the effective confining potential, and will not induce additional relaxations. However, strongly localized surface traps should be avoided, since the nearby trap states locating between $|0\rangle$ and $|1\rangle$ would easily induce nonradiative transitions between the trap states and the logic states due to the lattice relaxation mechanism [20], thus breaking the coherence of the qubit states. We are pleased that the current technology seems able to control the density of trap states at the value of $\sim 10^{10} / \mathrm{cm}^{2}$, which is smaller than the density of QD numbers of $\sim 10^{12} / \mathrm{cm}^{2}$. Thus the problem might not be so severe as we imagine. Another concern may come from the tuning when implementing the controlledNOT gate. Though we have demonstrated that the conditional detuning energy of $\sim 1 \mathrm{meV}$ is large enough for the selective optical driving, in practice the environment may cause a certain complexity on this issue. However, provided that the environment or its reaction on qubits does not sensitively depend on the qubit states, the conditional detuning energy on the target qubit will not be washed out by the environment, since its effect can be included by an overall renormalization on the logic state levels.

We optimistically believe that the practical extrinsic difficulties can be eventually resolved with time, and the present proposal seems to be a hopeful candidate for quantum computing. At this stage, we suggest the experimental implementation of a single qubit based on a couple of QDs as proposed above. Without doubt, this minor progress will be extremely exciting, since so far, to our knowledge, there has been no experimental qubit realization based on QDs, and this might be an important step toward semiconductor quantum computing.

In summary, the central idea of our proposal is to build up a single qubit from two coupled quantum dots, and utilize the quantum cascade laser to perform the gate operations. We have demonstrated that the spatial separation of the two logic states can reduce the decoherence and improve the gating ratio remarkably; meanwhile it also plays a key role in implementing the universal quantum logic gates. For completeness, a scheme of quantum measurement has also been suggested. A more thorough analysis for many issues in practice is beyond the scope of this paper, and will be done in the future.
[1] D. P. DiVincenzo, Science 269, 255 (1995).

[2] A. Ekert and R. Josza, Rev. Mod. Phys. 68, 733 (1996).

[3] A. M. Steane, Rep. Prog. Phys. 61, 117 (1998).

[4] Q. A. Turchette, C. J. Hood, W. Lange, H. Mabuchi, and H. J. Kimble, Phys. Rev. Lett. 75, 4710 (1995).

[5] J. I. Cirac and P. Zoller, Phys. Rev. Lett. 74, 4091 (1995).
[6] N. A. Gershenfeld and I. L. Chuang, Science 275, 350 (1997).

[7] B. E. Kane, Nature (London) 393, 133 (1998).

[8] D. Loss and D. P. DiVincenzo, Phys. Rev. A 57, 120 (1998).

[9] Y. Makhlin, G. Schön, and A. Shnirman, Nature (London) 398, 305 (1999).

[10] D. V. Averin, Solid State Commun. 105, 659 (1998). 
[11] L. B. Ioffe, V. B. Geshkenbein, M. V. Feigelman, A. L. Fauchere, and G. Blatter, Nature (London) 398, 679 (1999).

[12] J. Faist, F. Capasso, D. L. Sivco, C. Sirtori, A. L. Hutchinson, and A. Y. Cho, Science 264, 553 (1994).

[13] U. Bockelman and G. Bastard, Phys. Rev. B 42, 8947 (1990).

[14] H. Benisty, C. M. Sotomayor-Torres, and C. Weisbuch, Phys. Rev. B 44, 10945 (1991).

[15] X. Q. Li, H. Nakayama, and Y. Arakawa, Phys. Rev. B 59,
5069 (1999).

[16] X. Q. Li and Y. Arakawa, Phys. Rev. B 57, 12285 (1998).

[17] S. A. Gurvitz, Phys. Rev. B 56, 15215 (1997).

[18] A. Shnirman and G. Schön, Phys. Rev. B 57, 15400 (1998).

[19] I. L. Aleiner, N. S. Wingreen, and Y. Meir, Phys. Rev. Lett. 79, 3740 (1997).

[20] X. Q. Li and Y. Arakawa, Phys. Rev. B 56, 10423 (1997). 